\title{
Wolf-Rayet Wind Models from Hydrodynamic Model Atmospheres
}

\section{Götz Gräfener and Wolf-Rainer Hamann}

Institut für Physik, Universität Potsdam, Am Neuen Palais 10, 14469 Potsdam, Germany

\begin{abstract}
We present a parameter study of WR-type mass loss, based on the PoWR hydrodynamic model atmospheres. These new models imply that optically thick WR-type winds are generally formed close to the Eddington limit. This is demonstrated for the case of hydrogen rich WNL stars, which turn out to be extremely massive, luminous stars with progenitor masses above $\approx 80 M_{\odot}$. We investigate the dependence of WR-type mass loss on various stellar parameters, including the metallicity $Z$. The results depend strongly on the $L / M$ ratio, the stellar temperature $T_{\star}$, and the assumed wind clumping. For high $L / M$ ratios, strong WR-type winds can be maintained down to very low $Z$. Even for primordial massive stars we predict considerable mass loss if their surfaces are self-enriched by primary elements.
\end{abstract}

Keywords. stars: abundances - stars: atmospheres - stars: mass loss - stars: Wolf-Rayet

\section{Optically thick stellar winds}

A characteristic property of Wolf-Rayet (WR) stars is their strong mass loss. Their wind momenta typically lie above the single scattering limit $\left(\dot{M} v_{\infty}>L / c\right)$, raising the question if WR winds are driven by radiation.

In case of radiative driving, photons need to be absorbed and re-emitted more than once to achieve the large wind momenta. A direct consequence of this is that the fluxmean optical depth $\tau_{W}$ of the wind needs to be large. Photons are scattered on average $\tau_{W}^{2}$ times before they leave the wind. Because they perform a random walk they transfer a momentum of $\tau_{W} L / c$ per time interval to the wind. The resulting wind momentum is of the same order of magnitude, i.e., $\dot{M} v_{\infty} \approx \tau_{W} L / c$. Actually the wind momentum is slightly lower because part of the radiative momentum is used to overcome the gravitational potential of the star (see, e.g., Sect. 7.2.2 in Lamers \& Cassinelli 1999, for an exact derivation).

The large wind optical depth $\tau_{W}$ is also responsible for the spectral appearance of WR stars. For large $\tau_{W}$ the ionizing radiation from the static layers is absorbed within the wind. This leads to strong recombination, and via recombination cascades, to the observed WR emission line spectra. The fact that WR emission lines are observed together with large wind momenta is thus a hint that $\tau_{W}$ is large for WR stars, and that their winds are radiatively driven.

To generate large $\tau_{W}$ a large flux-mean opacity is needed directly above the sonic point, in combination with a large density scale height $H_{\rho}$. We thus expect WR-type mass loss to occur 1) close to the Eddington limit when $H_{\rho}$ becomes large, and 2) in temperature regimes where the sonic point is located close to the Fe-opacity peaks (see also Nugis \& Lamers 2002). 


\section{WR wind models from hydrodynamic model atmospheres}

The Potsdam Wolf-Rayet (PoWR) models combine fully line-blanketed non-LTE model atmospheres with the equations of hydrodynamics (for details see Gräfener \& Hamann 2005; Hamann \& Gräfener 2003; Koesterke et al. 2002; Gräfener et al. 2002). The wind structure $(\rho(r)$ and $v(r))$ and the temperature structure $T(r)$ are computed consistently with the full set of non-LTE populations, and the radiation field in the co-moving frame $(\mathrm{CMF})$. In contrast to other approaches, the radiative wind acceleration $a_{\text {rad }}$ is obtained by direct integration

$$
a_{\mathrm{rad}}=\frac{1}{c} \int \chi_{\nu} F_{\nu} \mathrm{d} \nu
$$

instead of making use of the Sobolev approximation. In this way, complex processes, e.g., due to the strong line overlap or the redistribution of radiation, are automatically included. Moreover, the models take small-scale wind clumping into account. The models describe the conditions in optically thick WR atmospheres in a realistic manner, and provide synthetic spectra, i.e. they allow for a direct comparison with observations.

\subsection{Results for different WR subtypes}

Utilizing the PoWR models, we have obtained the first fully self-consistent WR wind model for a WC star of early subtype (Gräfener \& Hamann 2005). Moreover, we have examined the mass loss of late-type WN stars and its dependence on metallicity (Gräfener \& Hamann 2006, 2008).

According to our models, WR-type mass loss is triggered by the proximity to the Eddington limit. The sonic-point temperatures lie in the expected range for optically thick winds $(\approx 200 \mathrm{kK}$ for early WC subtypes, corresponding to the hot Fe-peak; $30-45 \mathrm{kK}$ for late WN subtypes, corresponding to the cool Fe-peak). Because of this, we predict two distinct regimes of stellar core temperatures for which strong WR-type mass loss occurs. For early WC subtypes very high core temperatures of the order of $T_{\star} \approx 140 \mathrm{kK}$ are required (Gräfener \& Hamann 2005). WNL subtypes can be reproduced successfully for cooler temperatures, in the range $T_{\star}=30-50 \mathrm{kK}$ (Gräfener \& Hamann 2006, 2008). For intermediate values of $T_{\star}$ our models do not provide enough radiative force in the deep atmospheric layers, i.e., we do not obtain a stationary wind solution. In this regime pulsations might play an important role (e.g., strange mode pulsations as proposed by Glatzel \& Kaltschmidt 2002).

\subsection{The role of wind clumping}

An important feature of WR-type winds is their dependence on the wind clumping factor $D(r)$ (see Hamann \& Koesterke 1998). The wind acceleration roughly scales as $a_{\text {rad }} \propto$ $\sqrt{D}$. This effect is caused by the dominance of recombination processes in WR winds. An increase of $D$ increases the mean $\left\langle\rho^{2}\right\rangle$, and thus mimics a denser wind with a larger mean opacity. For models of early-type WR stars we usually have to choose relatively large clumping factors around $D=50$ to reproduce the observed terminal wind velocities. As observational estimates lie more in the range of $D=10$, our models thus might underestimate $a_{\mathrm{rad}}$ by roughly a factor of two. This could possibly reflect the lack of trace elements like $\mathrm{Ne}, \mathrm{Ar}, \mathrm{Mg}$, or $\mathrm{Ca}$ in our models.

\section{The most massive stars: H-rich WNL stars}

The high L/M ratios which are required to maintain WR-type winds can in principle be reached in different ways. He-burning stars have high L/M ratios because of their large 


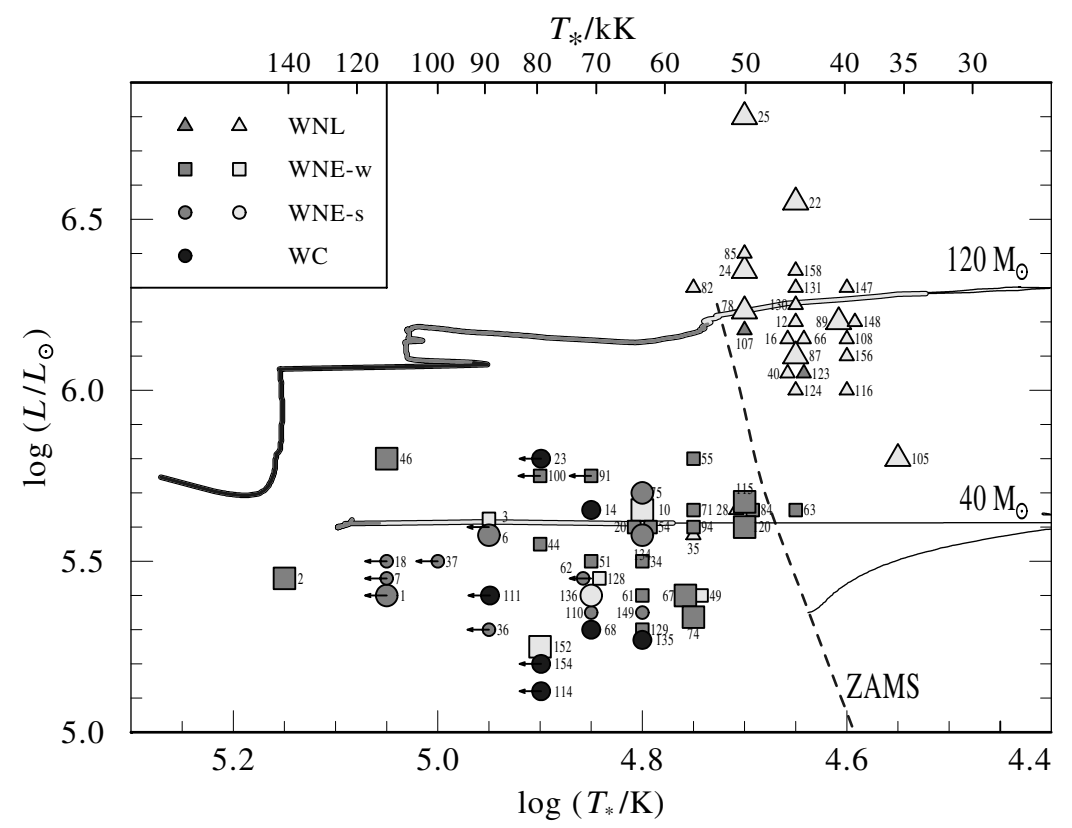

Figure 1. Spectral analyses of galactic WR stars with line-blanketed PoWR models, according to Hamann et al. (2006) and Barniske et al. (2006): symbols in light grey denote H-rich WN stars, whereas $\mathrm{H}$-free objects are indicated in dark grey. WC stars are indicated in black. For objects with large symbols distance estimates are available (van der Hucht 2001), whereas objects with small symbols are calibrated by their spectral subtype. Evolutionary tracks for non-rotating massive stars (Meynet \& Maeder 2003) are shown for comparison.

mean molecular weight. H-burning stars, on the other hand, can in principle reach high Eddington factors if their masses are extremely high. In the following we will show that this is indeed the case for H-rich WNL stars in young stellar clusters. Our models imply very high masses for these objects, in line with measurements of very high stellar masses in WNL binary systems (e.g., Rauw et al. 1996; Schweickhardt et al. 1999; Bonanos et al. 2004; Moffat et al. 2007).

\subsection{Spectral analyses of galactic WR stars}

A comprehensive study of galactic WR stars, based on spectral analyses with lineblanketed PoWR models (Hamann et al. 2006; Barniske et al. 2006, respectively for WN and WC stas) reveal a bimodal WR subtype distribution in the HRD (Fig. 1). Hrich WNL stars are located to the right of the ZAMS with luminosities above $10^{6} L_{\odot}$, while the mostly $\mathrm{H}$-free early- to intermediate $\mathrm{WN}$ subtypes, as well as the WC stars, have lower luminosities and hotter temperatures.

Although a part of the WNL stars with unknown distances (small symbols in Fig. 1) might actually belong to the group with lower luminosities, the dichotomy implies that H-rich WNL stars are the descendants of very massive stars, possibly still in the phase of central H-burning, whereas the earlier subtypes (including WC stars) are more evolved, less massive, He-burning objects.

\subsection{Wind models for $H$-rich WNL stars}

Gräfener \& Hamann (2008) investigate the properties of luminous, H-rich WNL stars by means of grid computations with hydrodynamic PoWR models. The most important conclusion from this work is that WR-type mass loss is triggered by the proximity to the 


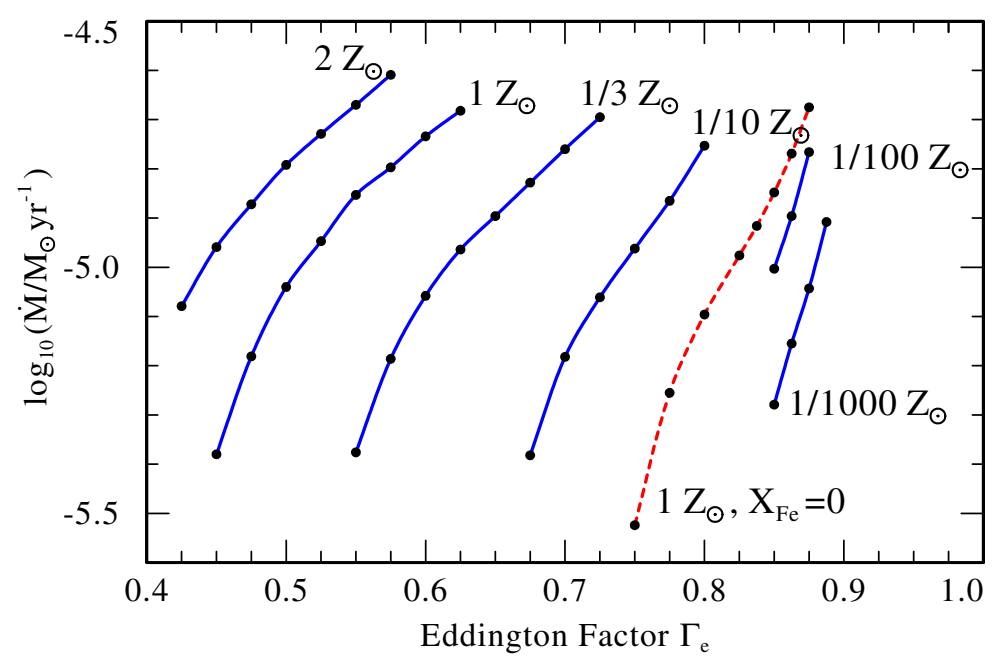

Figure 2. Wind models for WNL stars: mass loss rates from our hydrodynamic models are plotted vs. the Eddington factor $\Gamma_{\mathrm{e}}$. The stellar parameters are $L_{\star}=10^{6.3} L_{\odot}, T_{\star}=45 \mathrm{kK}$, and $X_{\mathrm{H}=0.4}$. The variation of $\Gamma_{\mathrm{e}}$ thus reflects a variation of the stellar mass. Solid curves indicate model series for different metallicities $Z$. The dashed line indicates models with zero Fe-abundance but solar-like CNO, resembling the composition of self-enriched very metal-poor stars (see Meynet et al. 2006).

Eddington limit (i.e., $\Gamma_{\mathrm{e}} \equiv \chi_{\mathrm{e}} L_{\star} / 4 \pi c G M_{\star}$ approaching unity), or equivalently, by high $L / M$ ratios. In Fig. 2 we show our results for H-rich WNL stars with a fixed luminosity of $10^{6.3} L_{\odot}$, illustrating the strong dependence of the mass loss on $\Gamma_{\mathrm{e}}$ and $Z$.

H-rich WNL stars typically have stellar temperatures in the range of $30-50 \mathrm{kK}$ and moderate wind optical depths $\left(\tau_{W} \approx 1\right)$. Their winds are initiated by the cool Fe-peak opacities. As can be seen in Fig. 2, the mass loss increases very rapidly when the stars approach the Eddington limit. However, the models are still away from the classical Eddington limit (i.e., $\Gamma_{\mathrm{e}}=1$ ), dependent on the adopted metallicity $Z$. This effect is caused by the influence of metal lines in the hydrostatic layers. The metal lines increase the flux mean opacity, and shift the 'true' Eddington limit, where the atmosphere actually becomes instable, towards lower values of $\Gamma_{\mathrm{e}}$, i.e., towards higher masses. Our solar $Z$ WNL models thus become WR stars already for $\Gamma_{\mathrm{e}} \approx 0.5$, a value which can be reached by very massive stars at the end of their main sequence evolution.

A more detailed analysis of the WN 7 component in WR 22, an eclipsing WR+O binary system in Car OB1, supports our results. Our models give a luminosity of $10^{6.3} L_{\odot}$, and a mass of $M_{\mathrm{WR}}=78 M_{\odot}$ for this object, in agreement with Rauw et al. (1996) who find $M_{\mathrm{WR}} \sin ^{3} i=72 \pm 3 M_{\odot}$ from the binary orbit (see Gräfener \& Hamann 2006, 2008). Such high masses imply that H-rich WNL stars are still in the phase of central H-burning, supporting an evolutionary sequence of the form $\mathrm{O} \rightarrow \mathrm{WNL}$ (H-rich) $\rightarrow \mathrm{LBV}$ $\rightarrow \mathrm{WN}$ (H-poor) $\rightarrow$ WC for very massive stars, as originally proposed by Langer et al. (1994).

\subsection{WNL stars: mass loss properties}

In Fig. 3 we show the temperature dependence of our WNL models. With $\dot{M} \propto T_{\star}^{-3.5}$ the mass loss nearly scales with the size of the stellar surface, a behavior which is not expected from the standard theory of radiatively driven winds (Castor et al. 1975, CAK). Moreover, we find a remarkably weak dependence of $\dot{M}$ on the luminosity (for fixed values $\Gamma_{\mathrm{e}}$ and $\left.T_{\star}\right)$. As pointed out by Gräfener \& Hamann (2008) these peculiar properties can 


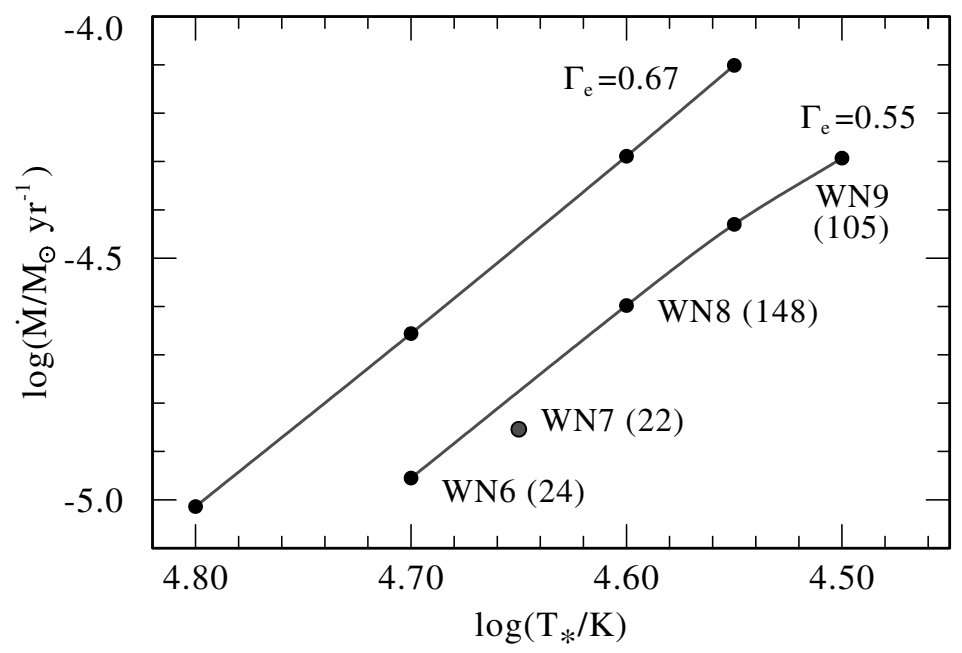

Figure 3. Wind models for galactic WNL stars: mass loss rates for different stellar temperatures $T_{\star}$ and Eddington parameters $\Gamma_{\mathrm{e}}$. The models reproduce the observed spectral sequence from WN 6 to WN 9. The corresponding spectral subtypes are indicated together with WR numbers of specific galactic objects (according to van der Hucht 2001, in brackets).

be understood within the theory of optically thick stellar winds (e.g., Nugis \& Lamers 2002). They are caused by the necessity to reach a specific temperatures at the sonic point, which demand for specific wind optical depths $\tau_{W}$.

\subsection{Metallicity dependence \& the first WN stars}

Our models in Fig. 2 show a $Z$-dependence where the onset of WR-type mass loss, in terms of the Eddington factor $\Gamma_{e}$, depends strongly on $Z$. This means that, on average, WR mass loss declines for lower $Z$. However, for larger Eddington factors our models predict equally strong WR mass loss rates over the whole range of metallicities, from 1/1000$2 Z_{\odot}$. Notably, we even obtain strong WR mass loss for iron-free models with solarlike CNO abundances (the dashed red line in Fig. 2). The composition of these models resembles extremely metal-poor stars which are self-enriched with primary nitrogen. The occurrence of such stars has been discussed, e.g., by Meynet et al. (2006). Our models thus predict an efficient mass loss mechanism for initially metal-poor objects (the first WN stars) which could play an important role in the enrichment of the early ISM.

\subsection{Mass determinations: the stellar population in the Arches cluster}

From IR photometry of the brightest stars in the Arches cluster, Figer (2005) has deduced a general upper mass limit for stars of $\sim 150 M_{\odot}$. In a recent work Martins et al. (2008) have determined the stellar parameters of these objects by means of detailed spectral analyses. The stars turned out to be extremely luminous WNL and Of stars, exactly matching the properties of our WNL grid models. Here we apply our results in the form of a parameterized mass loss recipe (of the form $\dot{M}\left(L_{\star}, T_{\star}, \Gamma_{\mathrm{e}}, Z, X_{\mathrm{H}}\right.$ ), see Gräfener \& Hamann 2008), to estimate the present masses of these objects.

In Fig. 4 we show our results for an adopted solar metallicity (we have performed computations for $Z_{\odot}$ and $2 Z_{\odot}$, giving qualitatively similar results). Notably, we find two stellar populations, namely objects with present masses below $\sim 100 M_{\odot}$ which are still on the main sequence, and chemically more evolved objects in the range of $30-100 M_{\odot}$ which might partly already burn helium, and seem to originate from more massive stars. The initial masses of the first group seem to lie in a range up to $130 M_{\odot}$, lifting the 


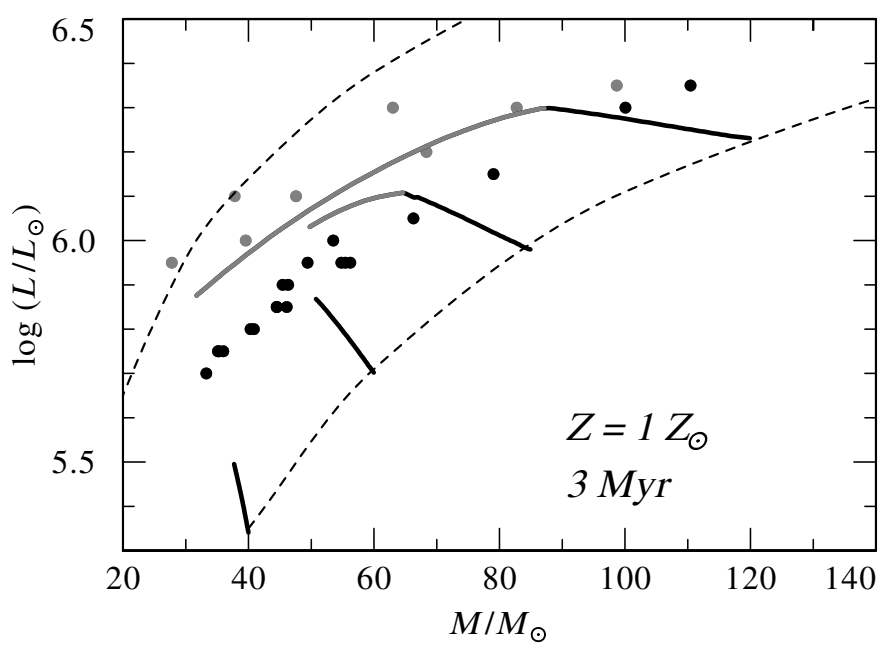

Figure 4. The most massive stars in the Arches cluster: plotted are luminosities taken from Martins et al. (2008), vs. present stellar masses, as derived from our wind models, for the brightest stars in the Arches cluster. We detect two populations with different L/M ratios, namely main sequence objects with solar hydrogen content (black dots), and more evolved He-rich objects (grey dots). We compare our results with rotating stellar models with an age of $3 \mathrm{Myr}$, according to Meynet \& Maeder (2003). The dashed lines indicate the ZAMS, and the He-MS for very massive stars (Ishii et al. 1999).

second group significantly above this value. If we adopt a larger value for $Z$ our mass estimates would even rise.

\section{References}

Barniske, A., Hamann, W.-R., \& Gräfener, G. 2006, in: H. J. G. L. M. Lamers, N. Langer, T. Nugis, \& K. Annuk (eds.), Stellar Evolution at Low Metallicity: Mass Loss, Explosions, Cosmology (San Francisco: ASP) ASP Conf. Ser., 353, 243

Bonanos, A. Z., Stanek, K. Z., Udalski, A., et al. 2004, ApJL, 611, L33

Castor, J. I., Abbott, D. C., \& Klein, R. I. 1975, ApJ, 195, 157

Figer, D. F. 2005, Nature, 434, 192

Glatzel, W., \& Kaltschmidt, H. O. 2002, MNRAS, 337, 743

Gräfener, G., \& Hamann, W.-R. 2005, A $\mho A$, 432, 633

Gräfener, G., \& Hamann, W.-R. 2006, in: H. J. G. L.M. Lamers, N. Langer, T. Nugis, \& K. Annuk (eds.), Stellar Evolution at Low Metallicity: Mass Loss, Explosions, Cosmology (San Francisco: ASP) ASP Conf. Ser., 353, 171

Gräfener, G., \& Hamann, W.-R. 2008, A\&BA, 482, 945

Gräfener, G., Koesterke, L., \& Hamann, W.-R. 2002, A\&SA, 387, 244

Hamann, W.-R., \& Gräfener, G. 2003, A\& A, 410, 993

Hamann, W.-R., Gräfener, G., \& Liermann, A. 2006, A\&SA, 457, 1015

Hamann, W.-R., \& Koesterke, L. 1998, A\&A, 335, 1003

Ishii, M., Ueno, M., \& Kato, M. 1999, PASJ, 51, 417

Koesterke, L., Hamann, W.-R., \& Gräfener, G. 2002, A\& A, 384, 562

Lamers, H. J. G. L. M., \& Cassinelli, J. P. 1999, Introduction to Stellar Winds (Cambridge: Cambridge University Press)

Langer, N., Hamann, W.-R., Lennon, M., et al. 1994, A\&BA, 290, 819

Martins, F., Hillier, D. J., Paumard, T., et al. 2008, A\&A A, 478, 219

Meynet, G., Ekström, S., \& Maeder, A. 2006, A\& A, 447, 623

Meynet, G., \& Maeder, A. 2003, A\&A, 404, 975

Moffat, A. F. J., Schnurr, O., Chené, A.-N., et al. 2007, Highlights of Astronomy, 14, 197 
Nugis, T., \& Lamers, H. J. G. L. M. 2002, A\&AA, 389, 162

Rauw, G., Vreux, J.-M., Gosset, E., et al. 1996, A\&A, 306, 771

Schweickhardt, J., Schmutz, W., Stahl, O., et al. 1999, A\&A, 347, 127

van der Hucht, K. A. 2001, New Astronomy Review, 45, 135

\section{Discussion}

NAJARro: The Geneva models with $2 Z_{\odot}$ you have used to explain the Arches cluster have indeed four times the Asplund's oxygen abundance and not twice, which is basically the value derived in the literature. Therefore the use of $2 Z_{\odot}$ models is not coherent with current estimates of the CNO content in the Arches.

GRÄFENER: The role of $Z$ in evolutionary models is that it controls the stellar mass loss rates. These are dominated by the Fe abundance. The Fe abundance however changed only slightly in Asplund's revision. So, concerning the tracks in the HRD, $2 Z_{\odot}$ remains $2 Z_{\odot}$. Moreover I have shown that my derived masses are in agreement with $2 Z_{\odot}$ tracks at an age of $2 \mathrm{Myr}$, and with $1 Z_{\odot}$ tracks at $3 \mathrm{Myr}$.

GAYLEY: Using Fe bumps to explain WR mass-loss rates seems to suffer somewhat from the problem of "having your cake and eating it too". If extreme temperature sensitivity in the opacity is crucial for understanding high mass-loss, why don't you have a problem with the wind stagnating as the temperature drops with radius in an optically thick wind?

GRÄFENER: This problem in fact exists for the winds of hot He-stars, but not for the H-rich WNL stars. The latter are driven by the cool Fe-peak alone. Our hot WCE models, on the other hand, with core temperatures of $140 \mathrm{kK}$ are initially driven by the hot Fepeak, and there are difficulties to overcome the gap between the two Fe-peaks. In our models we do this by increasing the clumping factor in this region. For slightly cooler stars the problem might be more severe. In reality the WCL stars even seem to form a second pseudo-photosphere because their initial wind stalls (see Petrovic et al., 2006, $A \& A, 450,219)$.

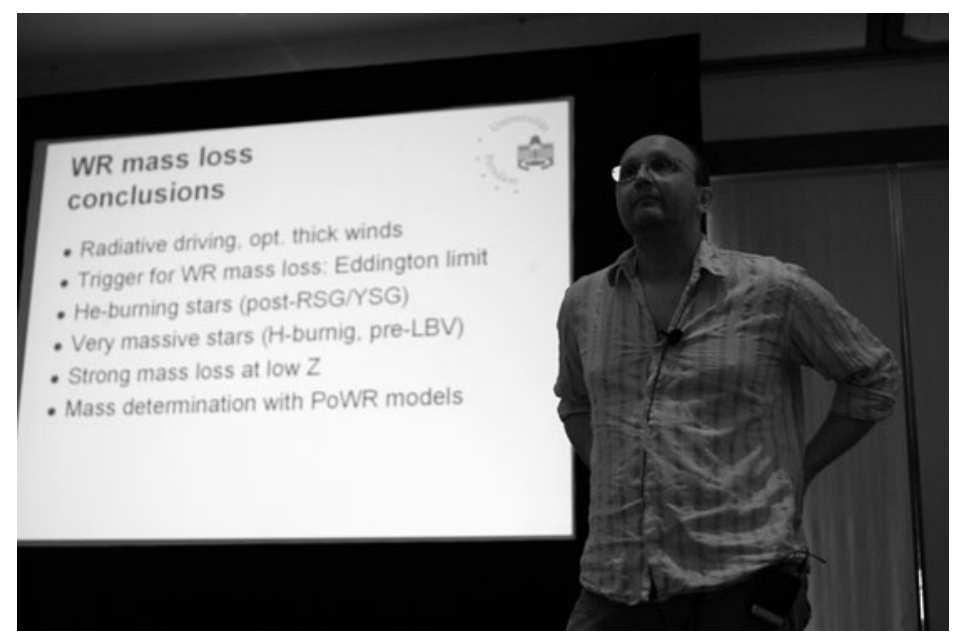

Götz Gräfener. 


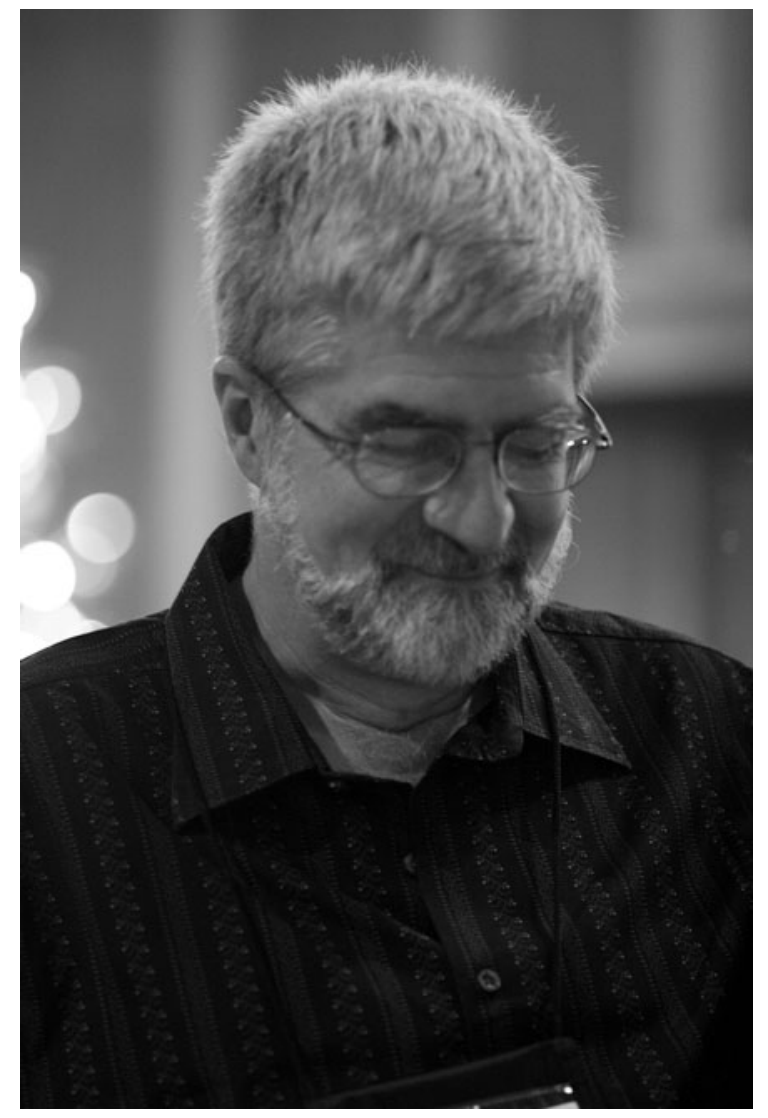

Philippe Eenens.

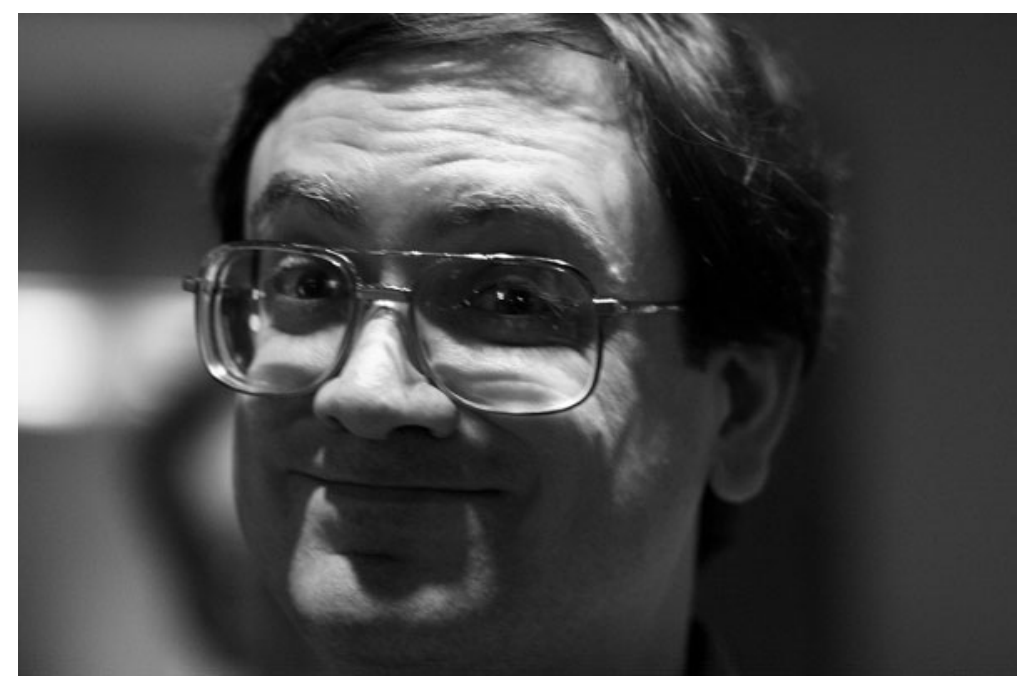

Steven Cranmer. 